\title{
China y el Mercosur: oportunidades en el desarrollo de sus relaciones
}

China and the Mercosur: Opportunities in the Development of Its Relations

Joaquín Andrés Gallego Mendaña ${ }^{1}$

\section{Resumen:}

Desde el comienzo de la década de 1990, los países de América del Sur notaron la importancia de las alianzas regionales. Como resultado de este nuevo pensamiento, algunos de ellos decidieron crear un bloque de integración: el Mercado Común del Sur (Mercosur), que nació oficialmente en 1991. Actualmente, Mercosur representa los intereses políticos y económicos de Argentina, Brasil, Paraguay, Uruguay y Venezuela, cuya población supera los 295 millones de personas.

Este proceso se convirtió en un pilar para la integración y cooperación comercial de América del Sur, cuyos resultados no habían sido vistos anteriormente en la región. De igual forma, se consolidó como un interlocutor para el acercamiento entre los líderes de los países para el desarrollo de una agenda común. Sin embargo, una gran cantidad de factores internos y externos han impedido al Mercosur alcanzar su máximo potencial.

Por otra parte, en este nuevo milenio, China ha emergido como un importante poder económico y comercial, de gran relevancia en la arena internacional. Es por ello que los países de América del Sur han tomado nota de su espectacular surgimiento económico y de su importancia en la creación de instituciones que están moldeando un nuevo mundo multipolar.

\footnotetext{
${ }^{1}$ Abogado argentino, mágister en Derecho civil y comercial chino. Actualmente es candidato a doctor por la Universidad de Renmin, China, e investigador del Centro de Estudios Latinoamericanos de la misma institución. Contacto:jogallego@lafalaw.com
} 
En este marco, el Mercosur está listo para interactuar con esta nación en una relación de largo plazo, que traiga beneficios para ambos actores.

Dichas oportunidades se pueden acotar en tres aspectos principales: el "Plan de Acción para la Integración de la Infraestructura Regional de América del Sur” (IIRSA) en relación con la Iniciativa china de "La Franja y la Ruta"; el Mercosur como un espacio de acercamiento entre Paraguay y China, y la Política de seguridad alimentaria en complementariedad con el mismo.

Palabras clave: China, América del Sur, Mercosur, Integración Regional, Oportunidades, Relaciones Internacionales.

\begin{abstract}
:
Since the beginning of the 1990s, the countries of South America have noticed the importance of regional alliances. As a result of this new way of thinking, some of them decided to create an integration block: The Southern Common Market (also named as "Mercosur"), which was officially born in 1991. Currently, Mercosur represents the political and economic interests of Argentina, Brazil, Paraguay, Uruguay and Venezuela, whose population exceeds 295 million people.
\end{abstract}

This process became a pillar for the integration and commercial cooperation of South America, whose results had not been seen before in the region. In the same way, it was consolidated as an interlocutor for the rapprochement between the leaders of the countries for the development of a common agenda. However, a large number of internal and external factors have prevented Mercosur from reaching its maximum potential.

On the other hand, in this new millennium, China has emerged as an important economic and commercial power, of great relevance in the international arena. That is why the countries of South America have taken note of its spectacular economic emergence and its importance in creating institutions that are shaping a new multipolar world. In this framework, Mercosur is ready to interact with this nation in a long-term relationship, which brings benefits for both actors. 
These opportunities can be identified in three main aspects: "The Action Plan for the Integration of the Regional Infrastructure of South America" (IIRSA) in relation to the "One Belt, One Road" Initiative; Mercosur as a space for rapprochement between Paraguay and China, and Food security policy in complementarity with it.

Keywords: China, South America, Mercosur, Regional Integration, Opportunities, International Relations.

\section{Introducción}

Desde el inicio de la Política de reforma y apertura, promovida por Deng Xiaoping en diciembre de 1978, el mundo ha experimentado una reconfiguración en el balance de poder. Los bloques regionales, tales como la Unión Europea, el Acuerdo de Libre Comercio de América del Norte (NAFTA), la Unión Africana, la Asociación de Naciones del Sudeste Asiático (ASEAN), entre otros, han jugado un rol clave en la construcción de un mundo multipolar.

Es en este nuevo escenario, que algunos países de América del Sur notaron la importancia de las alianzas regionales y decidieron crear un grupo regional, que actualmente representa los intereses políticos y económicos de más de 295 millones de personas en Argentina, Brasil, Paraguay, Uruguay y Venezuela. Como resultado de este nuevo razonamiento, el Mercado Común del Sur nació oficialmente en 1991, con la firma del Tratado de Asunción entre Argentina, Brasil, Uruguay y Paraguay; dando lugar a una alianza económica comercial entre estos países.

Tres años después, en 1994, el Protocolo de Ouro Preto delineó la estructura institucional del Mercosur. Hoy en día, esta unidad posee cinco miembros plenos -Argentina, Brasil, Paraguay, Uruguay y Venezuela ${ }^{2}-$, seis miembros asociados - Bolivia ${ }^{3}$, Chile, Colombia,

\footnotetext{
${ }^{2}$ Venezuela fue suspendida en 2016 por el no cumplimiento de sus obligaciones y nuevamente en 2017 por razones políticas; sin embargo, continúa siendo un miembro pleno del Mercosur bajo la categoría de "miembro suspendido". ${ }^{3}$ En 2012, Bolivia presentó formalmente su deseo de convertirse en un miembro pleno del Mercosur. Para que un país sea aceptado, es necesario que el congreso nacional de cada miembro del bloque apruebe dicha petición. Argentina, Uruguay, Paraguay y Venezuela ya han aprobado dicha solicitud, solo el congreso de Brasil no ha tratado el tema.
} 
Ecuador, Perú y Surinam- y tres países con estatus de observadores -México, Nueva Zelanda y Japón.

Esta entidad ha evolucionado de una unión aduanera a un espacio decisivo para aspectos relacionado con la migración, el trabajo, los intercambios culturales, la validación de diplomas; etc. De esta forma, el Mercosur se ha consolidado como una plataforma institucional para comerciar o invertir y, más allá de esa virtud, como un fenómeno cultural, histórico y político con vasto alcance en América Latina (AL) y el mundo. (Ferrer, 1996)

En este marco, es más útil concebirlo como un proyecto estratégico regional liderado por Estados, que proporciona a los países del Cono Sur una oportunidad para definir un rol independiente en la economía global emergente. En contraste, las políticas más relevantes de su organización se basan en decisiones tomadas por los gobernantes individuales y oficiales de las naciones que lo integran, más que en el comportamiento de los actores económicos. (Carranza, 2006) Sin embargo, desde su creación, una gran cantidad de factores internos y externos le han impedido alcanzar su máximo potencial.

Por otra parte, este milenio ha sido fundamental para constatar el ascenso de China como poder económico y comercial global. En tanto, los países de América del Sur han tomado nota de este espectacular despegue financiero y de la creciente influencia de dicho país en la creación de las instituciones que están moldeando el nuevo mundo multipolar. Hay que destacar que el actual presidente chino, Xi Jinping, que asumió el control del Estado en marzo de 2013, ha mostrado signos de que América Latina en general, y América del Sur en particular, son una prioridad emergente para su gobierno. Después de todo, América del Sur es el principal proveedor de materias primas que China necesita para continuar desarrollando sus industrias.

Por tanto, es en el Mercosur donde se encuentra la mayor reserva de petróleo del mundo -representada por Venezuela-, la segunda reserva más grande de agua potable -constituida por Argentina, Brasil y Paraguay-, y una de las reservas de litio más grandes del mundo -en las fronteras entre Argentina, Bolivia y Chile. Argentina y Brasil no sólo se encuentran entre los tres proveedores más grandes de porotos de soja -junto con Estados Unidos-, sino que también son miembros del G20, respaldo que ha genrado el reconocimiento por parte de China de su status como "socio estratégico integral" -incluyendo a Venezuela. 
Acualmente, tres de los cinco miembros del Mercosur gozan de dicho status. (Oviedo, 2006) Brasil, además, disfruta de una relación especial con China debido a su membresía en el grupo BRICS. ${ }^{4}$ De esta forma, el país asiático ha notado la importancia del Mercado Común del Sur, no sólo como un mero proveedor de materias primas, sino también como un mercado de consumo para sus productos y, aún más importante, como un aliado político internacional.

\section{Antecedentes históricos}

El Mercosur ha establecido diálogos directos o mecanismos de cooperación con varios países y bloques regionales, tales como: Estados Unidos, la Unión Europea, Corea del Sur, Japón, Rusia y China.

\section{1 "Diálogos Mercosur-China"}

La historia con éste último, se remonta a octubre de 1997, cuando una delegación encabezada por el embajador Aispirosa, del Uruguay -quien mantenía la presidencia pro tempore del bloque-, visitó China y sostuvo el primer diálogo con su contraparte asiática, conocido como "Diálogo Mercosur-China". Esta plataforma se convirtió en el canal por excelencia de comunicación entre ambos actores por un largo tiempo. Desde entonces, las dos partes han mantenido contacto regular en materia de asuntos políticos, económicos y de impacto internacional, que pudieran afectar los intereses mutuos.

El segundo diálogo tuvo lugar en Brasilia, el 9 de octubre de 1998. En aquella oportunidad, el entonces viceministro de relaciones internacionales de China, Yang Jiechi, y el asistente ministerial para comercio internacional, Gao Hucheng, se reunieron con el embajador de Brasil, Leonardo Maergesi, representante del Mercosur. Los involucrados acordaron mejorar las relaciones comerciales y promover la cooperación entre empresas en el plano tecnológico. Ese mismo día, un memorándum fue firmado con el compromiso de crear un foro en el cual inversores y empresas pudieran discutir sus principales intereses y preocupaciones.

El tercer diálogo se llevó a cabo el 18 de octubre del año 2000, en la ciudad de Beijing. Una vez más, un memorándum fue firmado entre ambas partes. Ese mismo día, el

\footnotetext{
${ }^{4}$ Asociación económica y comercial integrada por Brasil, Rusia, India, China y Sudáfrica.
} 
representante del Mercosur, el entonces viceministro brasileño de asuntos internacionales de Lima, se reunió con el vicepremier chino, Wen Jiabao. Al mismo tiempo, el viceministro de relaciones internacionales, Yang Jiechi, y el asistente ministerial de comercio internacional, Zhou Keren, mantuvieron una reunión de trabajo con la delegación del Mercosur, en la cual se intercambiaron visiones sobre las relaciones China-Mercosur. Ambos bandos remarcaron los desafíos existentes en el área de comercio internacional y la necesidad de explorar nuevas ideas sobre cómo mejorar la cooperación. (Ministerio de Relaciones Exteriores de China, 2017)

El cuarto diálogo se celebró en la ciudad de Montevideo, el 24 de septiembre del 2003. En aquella oportunidad, la presidencia del Mercosur estaba en manos del Uruguay, por lo que fue el viceministro uruguayo de relaciones internacionales, Guillermo Valles, quien se reunió con el viceministro chino de relaciones internacionales, Zhou Wenzhong. Durante este encuentro se debatió la importancia de fortalecer los intercambios y la cooperación entre Mercosur y China, especialmente en un mundo marcado por las profundas transformaciones a nivel regional y global. En tanto, la parte china manifestó un alto interés por mantener buenos canales de comunicación y cooperación relacionados con el comercio internacional; especialmente en lo referente a los asuntos a debatir en la Organización Mundial de Comercio (OMC), institución a la cual China había ingresado en el 2001. El objetivo de China durante dicho diálogo fue el de fortalecer los intereses de los países en vías de desarrollo dentro de la OMC.

Ambos viceministros estuvieron de acuerdo respecto al gigantesco potencial existente en las relaciones China-Mercosur, que aún no había sido aprovechado, y expresaron su compromiso en desarrollar dicho potencial para mejorar la vida de sus pueblos. Al finalizar la sesión, ambas partes convinieron fortalecer las relaciones comerciales, profundizar la cooperación en las áreas de agricultura y salud pública e incrementar el intercambio de personas, especialmente de académicos. También se discutió la posibilidad de crear un mecanismo para dar continuidad al "Diálogo Mercosur-China" y la posibilidad de firmar un acuerdo técnico de cooperación; sin embargo, ningún resultado fue alcanzado. En esa misma fecha, tuvo lugar el primer gran Foro de negocios entre China y el Mercosur. (Ministerio de Relaciones Exteriores de Uruguay, 2018). 
El quinto diálogo se llevó a cabo en junio de 2004, en la ciudad de Beijing. El entonces viceministro argentino de relaciones internacionales, Martín Redrado, encabezó una delegación bajo la presidencia pro tempore del bloque sudamericano. En dicho encuentro, se reunió con el viceministro chino de relaciones internacionales, Zhou Wenzhong. El representante del Mercosur y su contraparte china intercambiaron visiones en la negociación de un Tratado de Libre Comercio entre ambas regiones y decidieron comenzar un estudio de factibilidad sobre el mismo -el cual nunca se realizó. Este evento fue el último de su tipo y desde entonces dicho mecanismo ha caído en desuso. No obstante, los intercambios y la comunicación entre China y el Mercosur no se han interrumpido; por el contrario, se han configurado otras formas de cercanía.

Un dato no menor es que, durante la visita del presidente argentino, Mauricio Macri, a Beijing en 2017, el mandatario mencionó su voluntad y la del bloque sudamericano de reanudar el "Diálogo Mercosur-China". En esos momentos Argentina ejercía la presidencia pro tempore del bloque. Fue así que, en septiembre de 2018, se anunció que el día 18 de octubre de 2018 se reanudará dicho formato y se dará lugar al sexto diálogo en la ciudad de Montevideo. En esta oportunidad, los vicecancilleres de todos los países del bloque recibirán al viceministro de relaciones exteriores chino, Qing Gang. ${ }^{5}$

A pesar de que el último diálogo tuvo lugar en 2004, esto no implicó que la comunicación entre ambas partes cesara de manera indefinida. En cambio, un año después de dicho encuentro, en diciembre de 2005, Hu Jintao y el ministro de infraestructura Wang Guangdao participaron como observadores de la "29a Cumbre del Mercosur". Seis años después, en enero de 2011, el viceministro de asuntos exteriores, Li Jinzhang, felicitó al embajador brasileño Samuel Pinheiro Guimarães por su nombramiento por parte del Consejo del Mercado Común ${ }^{6}$ como Representante General del Mercosur. ${ }^{7}$ Dicha posición fue creada

\footnotetext{
${ }^{5}$ El Observador. (14/09/2018). "Mercosur reanudará el diálogo con China con una reunión de vicecancilleres en Montevideo". Obtenido de: https://www.elobservador.com.uy/nota/mercosur-reanudara-el-dialogo-con-china-conuna-reunion-de-vicecancilleres-en-montevideo-201891472623

${ }^{6}$ El Consejo del Mercado Común es el órgano superior del Mercosur, responsable de la conducción política y de la toma de decisiones con el fin de garantizar el cumplimiento de objetivos y términos establecidos. Dicho órgano está integrado por los miembros de los ministerios de relaciones exteriores y economía de todos los países miembros.

${ }^{7}$ La figura del alto representante del Mercosur fue creada en 2010, mediante la resolución del No. 63-10. Su principal función es la de promover y fortalecer la integración social del mismo. En julio de 2017, durante la Cumbre del
} 
durante la "Cumbre de Foz", de Iguazú, en 2010; esta figura tiene a cargo la articulación y la formulación de políticas, así como la representación de posiciones comunes dentro del bloque. También tiene la facultad de actuar en áreas como salud, educación, cultura y promoción.

\subsection{Un interés compartido}

En junio de 2012, China demostró nuevamente su interés por dialogar con Mercosur, durante la visita del premier Wen Jiabao a Argentina. En esa ocasión, el mandatario chino participó en una video conferencia con los presidentes de Argentina, Uruguay y Brasil, quienes asistieron a una reunión de alto nivel en Argentina. En dicho encuentro, ambas partes reiteraron su compromiso de profundizar la cooperación y el comercio. La particularidad de este plenaria fue la emisión de un "Comunicado conjunto para el mejoramiento de la cooperación comercial y económica entre China y el Mercosur”. Esta declaración enfatizó el compromiso de ambas partes para mejorar sus relaciones comerciales, poniendo la meta de que las mismas debían alcanzar los 200 mil millones de dólares para el año 2016. Al mismo tiempo, la declaración manifestó la postura compartida por ambos actores de oposición frente al proteccionismo de productos agrícolas por parte de los países desarrollados, y el reconocimiento de la inversión como pilar fundamental del crecimiento de los países en vías de desarrollo.

En agosto de 2012, el asistente ministerial para asuntos internacionales, Zhang Kunsheng, envió sus felicitaciones a Ivan Ramalho -funcionario brasileño- por su nombramiento como alto representante del Mercosur. (Ministerio de Relaciones Internacionales de China, 2013) En ese mismo año, en el mes de noviembre, una delegación del bloque participó en la "Feria Internacional de Alimentos" en la ciudad de Shanghái.

Posteriormenten, en octubre de 2015, el director general del departamento de asuntos latinoamericanos, Zhu Qingqiao, se reunió con el alto representante del Mercosur. Ambos funcionarios intercambiaron ideas en como mejorar el diálogo y la cooperación.

Mercosur que se realizó en Mendoza (Argentina), el bloque decidió la supresión de dicha figura bajo el fundamento de que ya existían instituciones con funciones similares. 
Más adelante, en diciembre de 2016, el representante de China para asuntos de América Latina, Yin Hengmin, se reunió con el representante uruguayo del Mercosur, Feyher, para abordar temas referidos con el mejoramiento de las relaciones China-América del Sur.

En cuanto al evento más reciente en el desarrollo de esta vinculación, se deben mencionar las intenciones de firmar un Tratado de Libre Comercio entre el bloque de América del Sur y China. Dichas intenciones fueron manifestadas durante la "Cumbre Presidencial del Mercosur", que se llevó a cabo en Paraguay, en junio de $2018 .^{8}$

Como se puede apreciar, los lazos que unen al Mercosur y a China no son nuevos. Pueden ser rastreados hasta inicios de 1997, cuando el primer "Diálogo Mercosur-China" se llevó a cabo. Este no es un dato menor si se tiene en cuenta que de los 27 años de existencia del Mercosur, en 21 de ellos han existido intercambios directos con China. Estos intercambios no solo han permitido a ambas partes mejorar sus relaciones, sino también crear conciencia sobre la existencia de desafíos y oportunidades en su trayectoria compartida.

Entre los desafíos, podemos nombrar la presencia de los Estados Unidos en la región, la existencia de diversos bloques regionales que desvían recursos de los países del Mercosur y la existencia de problemas internos dentro de los países miembros, que afectan el funcionamiento del bloque. Es por ello quer la presente investigación se concentrará en las oportunidades para el desarrollo de las relaciones Mercosur-China. En mayor medida en el ámbito de infraestructura, representado por el "Plan IIRSA" y "La Franja y la Ruta"; en el ámbito político, respecto a la utilización del Mercosur como un espacio de acercamiento entre Paraguay y China y en el ámbito de comercio agrícola, relacionado con la política de autosuficiencia alimentaria del gobierno chino y su complementariedad con el Mercosur.

\section{Oportunidades en el desarrollo de las relaciones Mercosur-China}

Desde su creación en 1991, el Mercosur ha atravesado distintas etapas, caracterizadas por diferentes regulaciones internas y por el modo de su relación con el mundo.

8 Soria, A. (12/06/2018). "El Mercosur evalúa ahora acercarse a China”. En La Nación. Obtenido de: https://www.lanacion.com.ar/2145268-el-mercosur-evalua-ahora-acercarse-a-china 
Se puede distinguir una primera etapa de 1991 a 1997, caracterizada por un alto grado de optimismo, no solo entre los miembros del bloque sino también por parte de los diferentes países observadores del resto del mundo. Durante este periodo, el proceso de democratización de los países integrantes finalmente se consolidó, dejando atrás un oscuro pasado, marcado por sangrientas dictaduras militares.

Esta etapa también se caracterizó por una mayor estabilidad macroeconómica, lo cual permitió al gobierno argentino superar su crisis hiperinflacionaria de 1991 y a Brasil recuperarse de un problema similar en 1993. A partir de entonces, se comenzó a observar un aumento en el comercio intrarregional de más de seis veces, a una tasa anual del $22 \%$, superando así al comercio extra regional. (Ferrer, 1996)

Una segunda etapa abarcó de 1997 hasta el 2015. Como consecuencia de la crisis asiática de 1997, la devaluación monetaria de Brasil en 1999 y la implosión de la economía argentina en 2001, el Mercosur ingresó en una fase de estancamiento, caracterizada por una falta de progreso en el proceso de integración de América del Sur y un aumento de las barreras comerciales entre los miembros del bloque. Es interesante notar que justamente durante este periodo, comenzaron los primeros contactos con China. Tal y como se mencionó anteriormente, el primer "Diálogo Mercosur-China" tuvo lugar en 1997, en la ciudad de Beijing, época en la que China estaba buscando activamente apoyo internacional para su ingreso a la OMC y en la que también exploraba nuevos mercados para la exportación de sus manufacturas de bajo costo.

Por lo tanto, la primera etapa del Mercosur fue más neoliberal y tuvo mayor afinidad con los principios establecidos en el "Consenso de Washington"; mientras que la segunda etapa fue más proteccionista, marcada por el rechazo abierto a los tratados de libre comercio con los países desarrollados. Un claro ejemplo de esta segunda etapa es el estrepitoso fracaso de la "Cuarta Cumbre de las Américas en Mar del Plata”, Argentina, en 2005. En esta cumbre, la idea de un "Tratado de Libre Comercio de las Américas" fue rotundamente declinada, cuyos principales opositores fueron los países que actualmente son miembros plenos del bloque, como es el caso de Argentina, Brasil y Venezuela.

Años más tarde, en 2015, comenzó una tercera etapa identificada por una pérdida de terreno por parte de los gobiernos de izquierda en Argentina, Brasil y Uruguay. Desde 
entonces, el Mercosur ha mostrado un renovado interés por alcanzar acuerdos comerciales con diferentes regiones del mundo, tales como la Unión Europea, Canadá, Japón, Corea del Sur y China. Esta tercera época también resalta por la reactivación del proceso de integración sudamericano mediante el acercamiento a otros bloques regionales, como la Alianza del Pacífico.

Las relaciones con China son consideradas de gran importancia por los líderes de todos los países miembros del Mercosur. Como consecuencia de esto, se han realizado numerosos intentos por interactuar a nivel país-país, lo cual ha generado resultados mediocres; esto ha sembrado la idea dentro del bloque de que debe crearse una agenda unificada entre los integrantes a la hora de interactuar con China.

Tomando en cuenta los intereses de ambos actores, manifestadas en el "Documento Blanco para Latinoamérica y el Caribe de 2008 y de 2016" y el "Comunicado Conjunto para el mejoramiento de la cooperación comercial y económica entre China y el Mercosur", de 2012, se pueden identificar ciertas áreas de oportunidad para el desarrollo de estos vínculos.

\subsection{El "Plan IIRSA" y "La Franja y la Ruta"}

A pesar de la falta de coordinación entre los países miembros del Mercosur, China posee una estrategia dirigida a este bloque, que se vio reflejada en el quinto "Diálogo Mercosur-China" y en el "Documento Blanco sobre Latinoamérica y el Caribe", de los años 2008 y 2016. En esos esfuerzos se puede constatar que la cooperación económica y comercial con China está cambiando de "ser espontánea" a "ser consciente", resultando en mayores requerimientos en la coordinación política e integración del desarrollo entre ambas partes. (ZHOU Zhiwei, YUE Yunxia, 2017)

En 2013, el presidente Xi Jinping anunció en Kazajistán su plan global de diseñar una nueva "Ruta de la Seda", con el objetivo de recrear la antigua "Ruta de la Seda", que comunicaba grupos de mercaderes desde lugares tan lejanos como Corea y Madrid. Conocida actualmente como “La Franja y la Ruta” (en chino como Yi Dai Yi Lu一带一路, en inglés One Belt, One Road-OBOR-), es un cinturón que comprende una vía terrestre y una marítima, que tiene el propósito de unir a China con otras regiones del mundo. (Frankopan, 2016) 
Es importante mencionar que América Latina está inculida de forma privilegiada dentro de estos planes, que guardan una reminiscencia de la ruta que unía Manila con Acapulco, de 1567 a 1815. En este tránsito, se trasladaban diversos productos, mayoritariamente seda, porcelana y té desde China; en el sentido inverso, se trasportaba plata proveniente de las minas mexicanas.

Este antecedente ha sido utilizado por los oficiales del gobierno chino, para proclamar que AL es una extensión natural de la nueva "Ruta de la Seda". Por ello, los países de la región han puesto su mirada en este horizonte, para lograr un mayor acercamiento con el país asiático. Una muestra de apoyo a "La Franja y la Ruta" ocurrió en 2017, cuando el presidente de Argentina, Mauricio Macri, y la presidenta de Chile, Michelle Bachelet, participaron en el "Foro de la Franja y la Ruta para la Cooperación Internacional", respaldando la idea de incluir a América del Sur en la propuesta de Xi Jinping.

Es claro que para los países del Mercosur, la importancia de China no solo recae en el hecho de que el país asiático es un gran consumidor de materias primas, sino también en el hecho de que se ha convertido en una gran fuente de capital global. Estas naciones ven a China como un actor importante de financiación, inversión y como un proveedor de préstamos con bajos intereses y pocas condiciones. (Lewit, 2015)

13 años antes de que el presidente chino anunciara el diseño de OBOR, en el año 2000, los 12 países que integran América del Sur presentaron el "Plan de Acción para la Integración de la Infraestructura Regional Suramericana", mejor conocido como IIRSA. Posteriormente, en 2011, se creó el Consejo Sudamericano de Infraestructura y Planeamiento (COSIPLAN) a través de la Unión de Naciones Sudamericanas (UNASUR). Este órgano es el responsable de coordinar e implementar la integración de la infraestructura en América del Sur; dentro de sus funciones, el COSIPLAN creó el "Plan Estratégico de Acción 2012-2022”.

De acuerdo al "Informe de la Cartera de Proyectos 2017" del COSIPLAN, actualmente existen 562 proyectos de integración, por un monto estimado de 198 mil millones de dólares, distribuidos en todo el territorio sudamericano. De estos 562 proyectos, 409 se encuentran bajo construcción por un monto de 150 mil millones de dólares. El 80\% de los proyectos son catalogados de "nivel nacional", mientras que el resto son "nivel multinacional". La gran mayoría de los proyectos del COSIPLAN (562) están dirigidos a la construcción de 
infraestructura con el objetivo de conectar diferentes ciudades. De los 562 proyectos, 258 son carreteras, 71 proyectos fluviales, 53 ferrocarriles, 35 proyectos marítimos, 24 aeropuertos, 14 transporte multimodal y el resto está relacionado con la producción de energía. (COSIPLAN, 2017)

Todos estos proyectos de infraestructura representan una excelente oportunidad para que el Mercosur se incluya dentro de OBOR. En este sentido, el plan de cooperación " $1+3+6$ ", anunciado por Xi Jinping en 2015, junto con el plan “3x3”, promovido por el premier Li Keqiang, deberían ser utilizados por el Mercado Común para mejorar su infraestructura.

De acuerdo con la Organización para la Cooperación y Desarrollo Económico (OCDE), se espera que del año 2016 al 2020 el crecimiento promedio anual de las importaciones de China de productos agrícolas, minerales y petróleo, provenientes del Mercosur, incrementen un $3.8 \%, 5.8 \%$ y $6.1 \%$, respectivamente. Mientras que, del 2021 al 2030, se avizora que estos números sean de 2\%, 2.8\% y 2.7\%. (XIE Wenze, 2017) Para que estas predicciones puedan convertirse en una realidad y promuevan un importante desarrollo de la región, es necesario que el Mercosur mejore su raquítica infraestructura. En tanto, para que las cantidades de materia primas mencionadas por la OCDE puedan ser exportadas a China, es necesario que éstas lleguen a un puerto para su posterior envío a Asia; es aquí donde la infraestructura ferroviaria juega un rol clave.

De acuerdo con el Banco Interamericano de Desarrollo, los costos logísticos en el bloque constituyen del $18 \%$ al $35 \%$ del valor final de sus productos, comparado con solo el $8 \%$ en los países miembros de la OCDE. Por ello, "La Franja y la Ruta" está en línea con esa necesidad de diversificar su infraestructura y sus sectores industriales. Bajo la "nueva normalidad" de la economía china, OBOR pone a disposición del Mercosur Plan de Acción para la Integración de la Infraestructura Regional Suramericana -y del resto de los países del mundo que sepan aprovecharlo- capital, tecnología y un surplus de capacidad laboral capaz de mejorar la infraestructura existente a través de inversiones. Por tanto, la falta de infraestructura adecuada es un obstáculo que debe ser superado para lograr una mayor integración del bloque sudamericano y al mismo tiempo una mayor competitividad de sus productos. (Panaia, 2014)

Por otra parte, dentro de los proyectos del COSIPLAN existe uno en específico de gran impacto para el Mercosur en el que China podría hacer importantes contribuciones; se trata 
del "Hub Mercosur-Chile”. Este proyecto fue planeado originalmente por los países miembros y era conocido como "Corredor central", pero las sucesivas crisis económicas fueron posponiendo el proyecto, el cual finalmente fue reflotado por el "Plan IIRS A" y el COSIPLAN. La idea del Hub es crear un corredor bioceánico que conecte las zonas más productivas localizadas en zonas costeras- con otras regiones a través de la construcción de autopistas, ferrocarriles, puertos, puentes, etc. El objetivo es trasladar de forma más eficiente mercaderías, personas y recursos dentro del bloque y al mismo tiempo conectar a todos los países integrantes con puertos localizados en el Océano Atlántico y Pacífico, para que puedan exportar a los mercados de Asia, África y Europa de manera más competitiva.

El Hub comprende cinco países ${ }^{9}$-Argentina, Brasil, Chile, Paraguay y Uruguay-, cubre una superficie de 3,2 millones de kilómetros cuadrados, con una población de 140 millones de personas -el $48 \%$ de todos los sudamericanos-e incluye 123 proyectos de infraestructura valuados en 54,6 mil millones de dólares. El área comprendida es la responsable de generar el 70\% de la actividad económica de América del Sur y su PIB es de 850 mil millones de dólares, de los cuales, el $85 \%$ proviene de Brasil y Argentina. (XIE Wenze, 2017)

Otro proyecto del COSIPLAN de gran impacto para el Mercosur, es la rehabilitación y construcción de cuatro ferrocarriles transcontinentales, que se extenderían de Este a Oeste, atravesando horizontalmente toda América del Sur, y otros dos que se extenderían desde la punta Norte hasta Cono Sur. De acuerdo a la Asociación Latinoamericana de Ferrocarriles (ALAF), América Latina posee casi 100,000 kilómetros de vías férreas, de los que alrededor de 60,000 kilómetros se encuentran en el territorio del Mercosur; siendo Argentina y Brasil los mayores propietarios de ferrocarriles. (ALAF, 2018)

La idea del COSIPLAN es usar las vías férreas ya existentes, rehabilitar aquellas que han caído en desuso y construir nuevas donde sea necesario, para conectar toda América del Sur a través de los ya mencionados trenes transcontinentales. La ruta de los cuatro trenes transcontinentales sería: Tren Transcontinental Campos (Brasil)-Bayovar (Perú); Tren

\footnotetext{
${ }^{9}$ El "Hub Mercosur-Chile" está integrado por los estados del sur de Brasil -Minas Gerais, Sao Paulo, Santa Catarina, and Rio Grande do Sul-, todos los estados del Uruguay, la región occidental de Paraguay, numerosas provincias de Argentina -Misiones, Corrientes, Entre Ríos, Buenos Aires, Santa Fe, Córdoba, La Pampa, San Luis, Mendoza y San Juan- y cuatro estados de Chile -Coquimbo, Valparaíso, Libertador O’Higgins y Santiago.
} 
Transcontinental Santos (Brasil)-Santa Cruz (Bolivia)-Ilo (Perú); Tren Transoceánico Paranagua (Brasil)-Asunción (Paraguay)-Chaco (Argentina)-Antofagasta (Chile), y Tren Transoceánico Buenos Aires (Argentina)-Valparaíso (Chile). Los dos ferrocarriles que atravesarían América del Sur de Norte a Sur serían: el Tren Río Grande (Brasil)-Belem (Brasil) y el Tren Buenos Aires (Argentina)-Santa Cruz (Bolivia). El 90\% de las vías férreas de este proyecto se encontrarían localizadas en el territorio del Mercosur.

Algunas de estas rutas ya existen y han caído en desuso, por lo que necesitan ser rehabilitadas. Tal es el caso del tren que une Buenos Aires (Argentina) y Santa Cruz (Bolivia), que actualmente transporta muy poca carga y cuyas trochas poseen diferentes medidas. Rn cambio, otras vías necesitan ser construidas; como es el caso del Tren Transcontinental que une Brasil con Perú. Justamente, respecto a este proyecto, el presidente Xi visitó Brasil en el año 2014 y junto a los presidentes de Brasil y Perú creó un equipo de trabajo ${ }^{10}$ para llevar adelante un estudio de factibilidad de dicho proyecto, que fue terminado en el $2016 .{ }^{11}$

En tanto, la carencia de infraestructura adecuada, como en el caso de la autopista San Pablo-Montevideo-Buenos Aires y el Túnel de Agua Negra ${ }^{12}$-en construcción-, ha creado cuellos de botella en las capacidades exportadoras del Mercosur. (Panaia, 2014) Por lo que estas capacidades se verían altamente beneficiadas con la consolidación de los proyectos del "Hub Mercosur-Chile" y de los ferrocarriles transcontinentales; pero la falta de infraestructura en el Mercosur se debe en gran parte a la falta de financiamiento y capital.

\footnotetext{
${ }^{10}$ Memorandum de entendimiento entre el Ministerio de Transporte de la República Federal de Brasil, 1. C. (2014). Consejo Empresarial Brasil-China. Obtenido de Consejo Empresarial Brasil-China: http://www.cebc.com.br

11 Agencia de noticias Xinhua. Accedido 12 de Agosto de 2018. Obtenido de http://www.xinhuanet.com/english/2015-05/20/c_134252888.htm

${ }^{12}$ Actualmente solo existen dos caminos aptos para el transporte de mercaderías entre Argentina y Chile a través de los Andes, estos son el Paso Jama y Cristo redentor. Sin embargo, ambos caminos son ocasionalmente cerrados debido a las condiciones meteorológicas. El Túnel de Agua Negra es un proyecto que fue anunciado en 2012 y que se encuentra bajo construcción por el cual se unirá Argentina y Chile a través de un túnel de 14 kms. de largo atravesando las Cordillera de los Andes. Una vez finalizado dicho túnel permitirá unir los puertos de San Pablo en Brasil con los de Valparaíso en Chile, conectando al mismo tiempo a Argentina, Uruguay y Paraguay.
} 
A la fecha, el Fondo para la Convergencia Estructural del Mercosur (FOCEM) solo ha sido capaz de sufragar un proyecto multimodalvaluado en 184 millones de dólares, de los cuales, el FOCEM aportó 83 millones y el gobierno uruguayo los 181 millones restantes. ${ }^{13}$

Por su cuenta, el gobierno chino, a través de la política de apertura al mundo y de la propuesta de "La Franja y la Ruta" ha ofrecido numerosos canales de financiación para América Latina, siendo los más importantes: el Fondo de Cooperación China-Brasil -15 mil millones de dólares-, el Fondo de Cooperación China-América Latina y el Caribe -10 mil mdd-, el Fondo para la Cooperación de la Capacidad Productiva China-América Latina -30 mil mdd-, el Préstamo Especial para Proyecto de Infraestructura en Latinoamérica -20 mil mdd- y el Préstamo Preferencial para América Latina -10 mil mdd. (XIE Wenze, 2017) Todos estos mecanismos de financiación combinados alcanzan la suma de 85 mil millones de dólares, cantidad que supera todos los proyectos ferroviarios contemplados en el plan IIRSA valuados en 47 mil millones de dólares. A esto hay que agregarle la creación del Banco Asiático de Inversión en Infraestructura (AIIB) del cual China, Brasil, Bolivia, Chile, Perú y Venezuela son miembros. (Zotttele y WEI Qian, 2017)

Teniendo en cuenta el "Plan Estratégico de Acción 2012-2022" del COSIPLAN y los enormes beneficios que podría aportar al Mercosur, el hecho de que China ofrece diversos fondos de financiación para infraestructura y préstamos preferenciales, el deseo de los países sudamericanos por desarrollar su infraestructura y el plan chino de OBOR, es difícil imaginar un área de cooperación con mayor potencial que el ya mencionado. Depende del Mercosur diseñar una agenda de trabajo junto a China para que la Iniciativa de "La Franja y la Ruta" y el desarrollo estratégico de infraestructura en América del Sur se lleven a cabo de forma benéfica para los actores involucrados.

\subsection{Mercosur como espacio de acercamiento entre Paraguay y China}

Desde su fundación en 1949, la República Popular China ha promovido constantemente la política de "Una sola China"; no solo como una forma de manifestar a la comunidad

\footnotetext{
${ }^{13}$ FOCEM. (s.f.). Recuperado el 20 de septiembre de 2018, de Fondo para la Convergencia Estructural del Mercosur: https://focem.mercosur.int/es/
} 
internacional que Taiwán es una parte vital e integral del territorio chino, sino también como una forma de interactuar individualmente con los diferentes países del mundo.

Las relaciones Mercosur-China no son la excepción a esta regla. El "Documento Blanco para Latinoamérica y el Caribe", emitido por el gobierno chino en 2008 y el "Documento Blanco para Latinoamérica y el Caribe", de 2016, mencionan que el principio de una sola China es un pilar político fundamental para Beijing a la hora de desarrollar sus relaciones con AL. Actualmente, de los 32 países integrantes de esta región, seis de ellos aún mantienen relaciones diplomáticas con Taiwán y no con Beijing. Estos países son: Belice, El Salvador, Guatemala, Honduras, Nicaragua y Paraguay. Este último es el único país del Mercosur y de toda América del Sur que no posee relaciones diplomáticas con China.

El último país del Mercosur en cambiar el reconocimiento diplomático de Taiwán a Beijing fue Uruguay, en 1988, motivado principalmente por intereses económicos. En aquel entonces, Uruguay buscaba incrementar la exportación de lana hacia China e incrementar el comercio bilateral en general. Hay varios factores que tienen importancia a la hora de mantener relaciones diplomáticas, ya sea con Taiwán o con Beijing: la política interna de cada país, diversas consideraciones geopolíticas, la presencia en las instituciones internacionales y las circunstancias regionales del momento. (Rodríguez, 2008) Los últimos tres factores aplican para países como Argentina y Brasil, ya que tienen un poder político y económico propio interno y bastante limitado a nivel internacional.

Pero en el caso de países territorialmente más pequeños como Paraguay y Uruguay, el peso económico de Taiwán o Pekín y las circunstancias regionales son los factores decisivos a la hora de elegir con cuál de los dos actores se mantendrán relaciones diplomáticas. Estos dos aspectos, aunados a la influencia de Argentina y Brasil, jugaron un rol fundamental en la decisión de Uruguay de reconocer diplomáticamente a China en el año 1988. Esto no es un dato menor si consideramos que, aún sin mantener relaciones diplomáticas, China compra una gran proporción de los porotos de soja producidos por Paraguay y que las importaciones chinas en Paraguay representan un cuarto del total. Por otro lado, mientras que el comercio entre 
Paraguay y Taiwán alcanzó los 50 millones de dólares en 2016, el comercio con China en el mismo periodo de tiempo creció hasta los 2,6 mil millones de dólares. ${ }^{14}$

Es por ello que la normalización de las relaciones indudablemente traería grandes beneficios comerciales para Paraguay y el Mercosur. El hecho de que Paraguay sea el único país que no posee relaciones diplomáticas con China, ha generado fricciones con los países del bloque. El Mercosur está interesado en el aumento de las relaciones comerciales con China e incluso en explorar la posibilidad de un tratado de libre comercio con el gigante asiático. Sin embargo, el hecho de que Paraguay no mantenga vínculos diplomáticos con China, es considerado como un obstáculo a la hora de llevar adelante las negociaciones.

Por otra parte, Argentina y Brasil han intentado persuadir a Paraguay de cambiar el estado de sus relaciones diplomáticas, pero todos los intentos han fracasado. (Oviedo, 2012) Durante la presidencia de Fernando Lugo, de 2008 a 2012, existieron altas expectativas de que un cambio de reconocimiento diplomático de Taipéi hacia Pekín podía ocurrir, sin embargo, esto nunca sucedió. Las expectativas surgieron en base al hecho que Lugo era miembro del Partido Colorado, partido político que ha estado promoviendo un mayor acercamiento entre Asunción y Pekín a través de la Asociación de Amistad Paraguay-China. Desde su creación la presidencia de dicha asociación, ha permanecido en cabeza de Diógenes Martínez, ex ministro de relaciones internacionales e histórico miembro del Partido Colorado. Dicha asociación se encuentra integrada por numerosas figuras que en algún momento mantuvieron o mantienen algún cargo en el gobierno paraguayo y todos ellos se han manifestado abiertamente en contra de los regalos y donaciones que Taiwán hace al país, por considerarlos inapropiados, arbitrarios y condicionantes de la política exterior paraguaya.

En la época en la que Argentina, Brasil, Uruguay y Venezuela establecieron relaciones diplomáticas con China, aún no existía el Mercosur. En esa época, los primeros acercamientos con China, que desencadenarían en el posterior reconocimiento diplomático del país asiático, debían realizarse con un alto grado de secretismo y solía consumir muchos recursos y energía concretar dichos espacios de diálogo. Algo muy similar continúa pasando hoy en día. Muchos

\footnotetext{
${ }^{14}$ Nations, U. (s.f.). International trade in goods and services based on UN Comtrade data. Obtenido de Department for International Trade and the Department for Business, Energy and Industrial Strategy in the UK: https://comtrade.un.org/labs/dit-trade-vis/?reporter=600\&partner=156\&type=C\&year=2016\&flow=2\&commodity
} 
de los países latinoamericanos que han establecido relaciones diplomáticas con China en los últimos años, como es el caso de Panamá -en el año 2017- y de República Dominicana -en 2018-, han debido mantener reuniones de alto nivel secretas y anunciar el cambio de relaciones diplomáticas de forma sorpresiva al mundo.

Ahora bien, instituciones regionales tales como el Mercosur o la Comunidad de Estados Latinoamericanos y Caribeños (CELAC) han jugado un rol importante en crear vínculos entre China y muchos países latinoamericanos. En el caso de Paraguay, el Mercosur ofrece un espacio propicio de diálogo, que no ha sido aprovechado, a pesar del gran interés del país asiático por consolidar su política de "Una sola China" en el continente Americano y de forma definitiva en América del Sur.

También, tiene la posibilidad de convertirse en un eficiente agente de comunicación entre Paraguay, China y el resto de los países de AL. Las principales razones para creer esto son: el "Diálogo Mercosur-China" tiene más de 20 años de existencia, trayectoria con una valiosa experiencia sobre cómo deben cooperar ambas regiones; además, la agenda de trabajo del Mercosur es más completa que la de otras organizaciones regionales. Los presidentes del bloque se reúnen al menos dos veces al año, así como sus diferentes grupos de trabajo, aún con mayor frecuencia.

Otra razón importante es que cuenta con la figura de la presidencia pro tempore, ejercida de manera anual y rotativa entre los diferentes miembros. El país con el mando en turno, está facultado no solo a impulsar la agenda de trabajo, sino también a representar al bloque como institución. Esto significa que China, en caso que así lo deseara, podría negociar directamente y de forma periódica con el Mercosur sin la necesidad de intermediarios. Otras organizaciones regionales no cuentan con este tipo de instituciones; de hecho, CELAC es solo un acuerdo político que reúne a los países de América Latina y el Caribe para promover la integración regional, pero no es una entidad política y no cuenta con un secretariado permanente, por lo que no se pueden tomar decisiones vinculantes para sus miembros. En cambio, el Mercado Común cuenta instituciones internas capaces de ejercer este tipo de funciones. ${ }^{15}$

\footnotetext{
${ }^{15}$ La Secretaría del Mercosur es una institución de carácter permanente creada por el Tratado de Asunción en 1991. Dentro de sus funciones encontramos: publicación de las normas creadas por el Mercosur, funciones administrativas para el funcionamiento del bloque, organización de todos los aspectos pertinentes a las cumbres, proveer de forma
} 
Con base en lo expuesto anteriormente, el Mercosur podría convertirse en un espacio de diálogo entre Paraguay y China, en el que se promoviera la coordinación y la cooperación entre ambos. Después de todo, Paraguay y China actualmente mantienen un intercambio comercial que crece año con año.

\subsection{Mercosur y la política de autosuficiencia alimentaria china}

Thomas Malthus fue uno de los primeros autores en plantear el tema de seguridad alimentaria. De acuerdo con Malthus (1798), si no existe control sobre el crecimiento de la población y ésta incrementa de forma sustancial, llegará un momento en el que los medios de producción no serán suficientes para alimentar a toda la población mundial. Según el teórico, una vez que toda la tierra fértil del mundo haya sido dividida entre la población, será imposible para la humanidad incrementar la producción de alimentos y satisfacer las necesidades de todos los habitantes del planeta.

Este problema, planteado por el autor de manera catastrófica, no ha pasado desapercibido para la comunidad internacional, la cual a través de diferentes organizaciones (FAO, UNCTAD, ONU, OEA, etc.) ha intentado combatir la falta de alimentos en el mundo. China, por su parte, siendo el país más poblado del mundo y uno con los de menor superficie cultivable, respondió a este dilema con una política nacional.

A partir de 1978, China comenzó a realizar una serie de reformas en el sector agrícola con el objetivo de ser capaz de proveer una fuente estable de granos y alimentos para su población. Desde entonces estableció que su gobierno debía ser capaz de producir alimentos para el 95\% de su población; esto significa que la autosuficiencia alimentaria constituye una política nacional de gran importancia. En América del Sur, un grupo de países ricos en recursos naturales, no solo son capaces de satisfacer las necesidades alimenticias de sus poblaciones, sino también capaces de exportar grandes cantidades de alimentos al mundo, contribuyendo a solucionar el problema de la seguridad alimentaria; se trata, por supuesto, de los países del Mercosur.

periódica información relevante a los países miembros, etc. Las funciones de la Secretaría fueron ampliadas en 2002 para incluir apoyo técnico y de consultoría por decisión del Consejo del Mercado Común No. 32/02. Dichas funciones fueron actualizadas en 2015, mediante la Decisión No. 15/15. 
El "Documento Blanco sobre Seguridad Alimentaria” de 1996, fue la primera publicación por el gobierno chino en la que se estableció la meta de alcanzar y mantener una tasa de autosuficiencia alimentaria del 95\%. ${ }^{16}$ Desde entonces, esta política se ha convertido en un pilar fundamental de la estrategia agrícola china. Sin embargo, el mismo documento reconoce la incapacidad china de alcanzar una tasa de $100 \%$ de autosuficiencia alimentaria. Es decir, el mismo gobierno chino reconoce que no sería posible lograr una producción de alimentos capaz de satisfacer a toda su población. Teniendo en cuenta esto, el Documento Blanco reconoció la necesidad de China de importar alimentos de otras partes del mundo para poder cubrir su demanda alimentaria. Para el año 2030, el gobierno chino calcula que la población alcanzará las 1,600 millones de personas, las cuales demandarán alrededor de 640 millones de toneladas de granos, con un estimado de $400 \mathrm{kgs}$. por individuo.

Esta Política de Seguridad Alimentaria no pasa desapercibida por el Partido Comunista Chino (PCC). En octubre de 2017, durante el "19Congreso Nacional del PCC", el presidente Xi Jinping remarcó la importancia que tiene la seguridad alimentaria en el mantenimiento de la estabilidad social. Xi también mencionó que deben redoblarse los esfuerzos para el mejoramiento y la modernización del sector agrícola, así como de las condiciones de vida de los agricultores. Respecto al tema, el presidente expresó: "debemos garantizar nuestra seguridad alimentaria para tener control de nuestra propia provisión de alimentos"17, además dijo, "vamos a iniciar una estrategia de seguridad alimentaria para que la gente pueda estar tranquila de lo que ponen en sus platos". ${ }^{18}$ Como se puede observar, el Partido no solo quiere asegurarse de que su pueblo tenga alimentos suficientes, sino que al mismo tiempo sean de buena calidad.

Gastón Fornés y Alan Butt Philip (2012) exponen que una de las motivaciones del contacto de China con América Latina es la de "alimentar a su gran población". (Fornes y Butt, 2012) Esta idea se encuentra reflejada en varios documentos oficiales, como el "Documento Blanco sobre Latinoamérica y el Caribe", del 2008 y 2016, y la "Declaración de Santiago" de la "II Reunión Ministerial del Foro CELAC-China", de 2018. En este último, en

\footnotetext{
${ }^{16}$ Beijing Information Office of the State Council of the People's Republic of China. (1996) China's Food Security Problem.

${ }^{17}$ Ibíd., p.28.

${ }^{18}$ Ibíd., p.43.
} 
la sección 6, tanto China como los países de AL manifiestan la importancia del sector agrícola como una de las ocho áreas de cooperación entre ambas regiones -también se mencionan política y seguridad, infraestructura y transporte, inversión, comercio y finanzas, industria, ciencia y tecnología, cooperación ambiental, intercambio cultural y cooperación en otras áreas. (CELAC, 2018)

El primer déficit comercial agrícola que China experimentó fue en 2008, por un total de 11 mil millones de dólares. ${ }^{19}$ Este fenómeno encendió las alarmas del gobierno y desde entonces China ha prestado especial atención a América Latina, como una región capaz de proveer una gran cantidad de materias primas y alimentos de manera constante y estable. Por esta razón, China comprendió que un mayor entendimiento con bloques regionales, tales como el Mercosur y la Alianza del Pacífico, es necesario para poder tener acceso a sus tierras ricas en recursos naturales.

China se ha convertido en un gran importador de materias primas de América Latina. Las importaciones de productos agrícolas del resto del mundo por parte de China se han incrementado de 15,500 millones de dólares, en 2003, hasta alcanzar 105,410 millones, en 2015. La región de AL es la mayor proveedora de productos agrícolas a ese país, con un 28,9\% del total importado, en 2015. Los Estados Unidos y Canadá le siguen con un 25,7\% y la región del Este de Asia se posiciona en tercer lugar, con un 16,5\% del total de los productos agrícolas importados. En último lugar se encuentra Europa, con un 10,5\%.

Es interesante notar que las importaciones chinas de productos agrícolas desde el Mercosur aumentaron de 9,000 millones de dólares, en 2003, a 73,500 millones, en 2017. Del total de las exportaciones de productos agrícolas que el Mercosur vende al mundo, China representa actualmente el $22 \%$ de las mismas. Por ello, no es extraño que Brasil sea el mayor proveedor de productos agrícolas de China; en 2017, el monto de importación fue de 24 mil millones de dólares. En segundo lugar se encuentra Estados Unidos, con 21 mil millones de dólares y en tercer lugar Canadá, con 6 mil millones. En 2017, por primera vez en la historia, Brasil superó a EE. UU. como el mayor proveedor de productos agrícolas del país asiático.

\footnotetext{
${ }^{19}$ Center, I. T. (s.f.). ITC Trade Map. Recuperado el 20 de septiembre de 2018, de Trade statistics for international business development: https://www.trademap.org/Index.aspx
} 
Detrás de Canadá se encuentra Australia -con 5,9 mil mdd-, Nueva Zelanda-con 5,6 mil mdd-, Indonesia -con 4,6 mil mdd-, Tailandia -con 4,4 mil mdd- y Argentina -con 3,5 mil mdd. En el puesto 16 se encuentra Uruguay, con 1,7 mil millones de productos agrícolas. Ni Paraguay ni Venezuela se encuentran entre los primeros 25 exportadores de este tipo de productos a China.

Estos números demuestran que el Mercosur juega un rol fundamental en la provisión de alimentos al mercado chino. Brasil es el mayor exportador de estos productos a China, mientras que Argentina se encuentra en el lugar 8 y Uruguay en el 16. Es decir, tres de los cinco miembros del bloque juegan un rol clave en el aprovisionamiento de alimentos por parte de China; por tanto, las exportaciones del Mercado Común se complementan con la política de autosuficiencia alimentaria del gobierno chino. Esta complementariedad debe ser usada como una oportunidad en el desarrollo de las relaciones.

El hecho de que el Mercosur pueda satisfacer la demanda de alimentos del gigante asiático de manera estable y confiable no es un dato menor para el gobierno chino, especialmente si se tiene en cuenta que el segundo mayor proveedor de alimentos a China es Estados Unidos con el que existe una guerra comercial- y el tercero es Canadá -fuerte aliado del anterior. Frente a la posibilidad de una súbita interrupción de los flujos de productos agrícolas entre China y Estados Unidos, solo ésta sería la única región del mundo capaz de producir la cantidad de alimentos que demanda la población china. Es decir, no solo posee las capacidades productivas para satisfacer esta demanda, sino que también comparte intereses; lo cual garantiza un flujo estable y constante de materias primas.

Con base en esta información, el Mercosur debería profundizar la cooperación en materia agrícola con China, para incrementar sus exportaciones al país asiático. Al mismo tiempo se podría agregar valor y tecnología a los productos exportados, impulsando el desarrollo de las economías partícipes. Por su parte, China podría consolidar su política de seguridad y autosuficiencia alimentaria con un socio que le garantice las cantidades de alimentos necesarias en todo momento. El rol de este bloque en la política de seguridad alimentaria china abre una ventana para un mayor acercamiento entre las dos regiones. 


\section{Conclusión}

El Mercosur fue fundado en 1991 y estableció sus primeros contactos directos con China en 1997, a través del "Diálogo Mercosur-China". Este intercambio se ha mantenido intercambios por 21 años. Durante este periodo, han surgido ciertas fricciones y obstáculos entre ambas partes, mismos podrían ser llamados "desafíos", que a la par son síntomas de un mayor acercamiento entre ambas regiones. Un mayor contacto trae consigo un mayor intercambio de ideas, que abre las puertas al debate sobre la manera en que las relaciones deben ser conducidas. Los intereses de las dos partes en la manera y/o en los sectores en los cuales existe potencial para un mejor y mayor desarrollo, se encuentran expresados en varios documentos mencionados con anterioridad y que ponen énfasis en tres áreas en específico: infraestructura, política y producción agrícola.

En cuanto a la infraestructura, es evidente la complementariedad del "Plan IIRSA" con “La Franja y la Ruta”. Los países del Mercosur tienen la necesidad de mejorar su infraestructura, por lo que mayor financiamiento y nuevas tecnologías son necesarios. Por su parte, China, a través de OBOR, ofrece numerosos canales de financiación y préstamos dirigidos a proyectos de infraestructura que ayuden a mejorar la conexión de dicho país con el resto del mundo.

En el área de política internacional, el Mercosur ofrece un espacio muy importante para que China consolide su política de "Una sola China" en América del Sur. Ofrece también una plataforma de acercamiento ideal entre China y Paraguay, el único país de Sudamérica que no posee relaciones diplomáticas con Beijing. El mejoramiento de las relaciones Paraguay-China, que no necesariamente signifique el reconocimiento de relaciones diplomáticas, tendría un impacto positivo en el desarrollo de los vínculos.

Respecto al sector agrícola, la necesidad de China de garantizar una cantidad significativa de granos y alimentos para alimentar a su población, abre una ventana para la cooperación entre el Mercosur y China. El país asiático ha establecido una política de autosuficiencia alimentaria mediante la cual se debe garantizar el acceso a alimentos para su población en todo momento y en la que los mercados internacionales juegan un rol importante, en la importación de productos agrícolas. El Mercado Común, como uno de los mayores 
exportadores de productos alimenticios agrícolas del mundo, se presenta como un socio con las capacidades de producción suficientes para satisfacer la demanda China y como un socio capaz de mantener un flujo constante de alimentos.

Estos tres sectores son prioritarios para el desarrollo de las relaciones Mercosur-China. Por tanto, es necesario que los países miembros del bloque sudamericano actúen de manera conjunta en la creación de una agenda común respecto a China y que, por su parte, el país asiático entienda la importancia del bloque regional como interlocutor de los intereses y deseos de Argentina, Brasil, Paraguay, Uruguay y Venezuela. Aprovechando las áreas mencionadas como un espacio para impulsar un mayor desarrollo de las relaciones entre ambos actores y la reanudación del mecanismo "Diálogo Mercosur-China", en octubre de 2018, otros aspectos podrían ser abordados posteriormente. El reflotamiento de este diálogo es un signo evidente de la importancia que dichas relaciones tienen tanto para China como para el Mercosur.

\section{Bibliografía:}

Carranza, M. E. (2006). “Clinging Together: Mercosur's Ambitious External Agenda, Its Internal Crisis, and the Future of Regional Economic Integration in South America". En Review of International Political Economy, Vol. 13, No. 5, pp. 802-829.

CELAC. (2018). Declaración de Santiago II Reunión Ministerial del Foro China-CELAC. Obtenido de http://www.cancilleria.gov.co/newsroom/news/declaracion-santiago-iireunion-ministerial-foro-celac-china-celac-china-trabajando

COSIPLAN. (2017). Informe de la Cartera de Proyectos del COSIPLAN 2017. Obtenido de http://www.iirsa.org/Document/Detail?id=4621

Ferrer, A. (1996). "Mercosur: trayectoria, situación actual y perspectivas". En Desarrollo Económico, Vol. 35, No. 140, pp. 563-583.

Fornés, G. \& Alan, B. P. (2012). The China-Latin America Axis: Emerging Markets and the Future of Globalization. Great Britain: CPI Antony Rowe, Chippenham and Eastbourne. 
Frankopan, P. (2016). The Silk Roads: A New History of the World. Great Britain: Bloomsbury Publishing Plc.

Lewit, A. (2015). Una nueva geopolítica: China-América Latina. Centro Estratégico Latinoamericano de Geopolítica. Obtenido de: http://www.celag.org/una-nuevageopolitica-china-america-latina/

Malthus, T. (1798). An Essay on the Principle of Population. London: Printed for J. Johnson, in St. Paul's Church-Yard.

Ministerio Uruguayo de Relaciones Internacionales. (2018). IV Encuentro del Dialogo Mercosur-China. Obtenido de http://www.mrree.gub.uy/frontend/page?1, inicio,ampliacionactualidad,O,es,0,PAG;C ONC;260;3;D;iv-encuentro-del-dialogo-mercosur-china;1;PAG;

Ministry of Foreign Affairs People's Republic of China. (2013). China's Foreign Affairs. Beijing: World Affairs Press.

Ministerio Chino de Relaciones Internacionales. (2017). Relaciones Mercosur-China. Obtenido http://www.fmprc.gov.cn/web/gjhdq_676201/gjhdqzz_681964/lhg_683262/zghgzz_6 83266/

Oviedo, E. D. (2006). “China: visión y practica de sus llamadas 'relaciones estratégicas””. En Estudios de Asia y África, Vol. 41, No. 3, 2006, pp. 385-404.

Oviedo, E. D. “Crisis del multilateralismo y auge de la diplomacia bilateral en la relación Mercosur-China". VI Reunión de la Red Estudios de América Latina y el Caribe sobre Asia-Pacifico.

Panaia, M. (2014). “Contribución al debate del uso y control del territorio”. En Marta P. (edit.), Mercosur la integración regional en tensión: Mercado de trabajo y fracturas en el Corredor Central. Buenos Aires: Editorial La Colmena.

Rodríguez, M.E. (2008). "La batalla diplomática de Beijing y Taipei en América Latina y el Caribe”. En Revista CIDOB d'Afers Internacionals, No.81, pp. 209-231. 
Smith, R. E. (2006). "Regional Integration Looking South to North: Mercosur and the Americas". En Perspectives on Work, Vol. 10, No. 1, pp. 37-40.

Xie Wenze. (2017). "The Cooperation of Infrastructure Construction between China and Latin America from the Perspective of the Belt and road Initiative: Taking the Cooperation of Railway Construction between China and South America as an Example". En Expanding the Belt and Road: A New Perspective on China-Latin America Integrated Cooperation. Beijing: Chinese Academy of Social Sciences, China Social Science Press.

Zhou Zhiwei \& Yue Yunxia. (2017). "Cooperation between China and Latin America: Overview, Opportunities and Challenges". En Expanding the Belt and Road: A New Perspective on China-Latin America Integrated Cooperation. Beijing: Chinese Academy of Social Sciences.

Zottele, A. C. (2010). “China y Argentina, políticas y consecuencias. Un recuento de los años recientes”. En Revista Orientando No. 1. Xalapa: Universidad Veracruzana.

Zottele, E. \& Wei Qian. (2017). "La Franja y la Ruta: Oportunidad para America Latina y búsqueda de un desarrollo sostenible”. En Orientando, Año 7, No.13. Xalapa: Universidad Veracruzana. 